\title{
On the nature and the role of the subsurface vesicles in the outer epithelial cells of the conjunctiva
}

\author{
P N DILLY \\ From the Department of Anatomy, St George's Hospital Medical School, Cranmer Terrace, Tooting, \\ London SW17 ORE
}

SUMMARY The layer of the tear film in contact with the conjunctiva is mucus. This mucus comes from two sources, the conjunctival goblet cells and the subsurface vesicles. These vesicles are found just below the surface of the conjunctival cells. They contain long chain mucus glycoprotein molecules that are joined to the vesicle membrane. The vesicles fuse with the surface membrane of the conjunctival cells and expose their mucus glycoprotein chains to the overlying mucus. Chemical and physical bonds between the two types of mucus help to bind the mucus layer to the conjunctiva. The vesicle membrane becomes incorporated in the cell membrane and supplies the membranes for the microvilli that cover the exposed surface.

The existence of membrane bound vesicles just below the cell membrane of the exposed surfaces of the outer layer of cells of the conjunctiva has been known for some time. They were first described by Wanko et al. in $1964^{\prime}$ from the perilimbal zone of the human conjunctiva. Takakusaki ${ }^{2}$ noted that the number of subsurface vesicles increased in vernal conjunctivitis and suggested that this was a pathological change. Srinivasan et al. ${ }^{3}$ found a similar increase in subsurface vesicles of the conjunctiva associated with a methyl methacrylate ocular prosthesis. Greiner et al. ${ }^{4}$ proposed that these vesicles were a second mucus secretory system of the human conjunctiva in addition to the well established conjunctival goblet cell source. Greiner et al. ${ }^{5}$ on the basis of toluidine blue staining and periodic acid Schiff (PAS) reactions demonstrated that the vesicle content was probably a mucoprotein in giant papillary conjunctivitis associated with hard contact lenses. He showed that the vesicle contents were discharged on to the surface of the eye. Greiner and Allansmith ${ }^{6}$ showed that the number of subsurface vesicles increased in contact lens wearers. Dilly and Mackie $^{7}$ showed that the number of vesicles was increased in the anaesthetic conjunctiva. Using a modified Thiéry's method ${ }^{8}$ as a specific stain for mucoprotein, they confirmed Greiner and colleagues $^{\text {sth }}$ conclusion as to the mucoprotein nature of the content of the subsurface vesicles.

Correspondence to Professor P N Dilly.

\section{Materials and methods}

With the informed consent of sufferers from external eye disease and persons with normal eyes human conjunctival biopsy specimens were taken from the lower lids with a $2 \mathrm{~mm}$ Elliott trephine. These specimens were fixed in glutaraldehyde. Similar larger biopsy specimens were taken immediately post mortem from a series of apparently normal eyes. These too were fixed in glutaraldehyde. They were processed for electron microscopy either by ruthenium red staining, tannic acid combined with ruthenium red, or by Thiéry's method. ${ }^{8}$ The specimens were embedded in 'Spurr' resin. Sections were cut with a Sorvall ultramicrotome, mounted on gold grids, and examined in a Phillips 301 transmission electron microscope.

\section{Results}

Besides these conditions, where there was an increase in number of subsurface vesicles,,-5 we have found in late stages of keratoconjunctivitis sicca a complete absence of vesicles.

The vesicles themselves varied in size from about $50 \mathrm{~nm}$ to $100 \mathrm{~nm}$ in diameter. They were usually circular or somewhat oval in profile. The vesicles might be more than one row deep beneath the cell membrane (Fig. 1).

Greiner et al. ${ }^{5}$ described the vesicles as single membrane bound. My results suggest that they are 
bounded by a double membrane very similar in structure to the cell surface membrane. This double membrane is easy to miss, because the nature of a spherical vesicle is that little of the surrounding membrane will be 'end on' to the electron beam and so be resolved as a unit membrane. However, by tilting the section in the electron beam until optimum alignment of the vesicle was achieved the unit membrane nature of the vesicle wall could be resolved (Fig. 2).

Specialised electron microscope staining for mucus glycoprotein by Thiéry's ${ }^{8}$ silver method showed that the subsurface vesicles contain a mucoprotein (Figs. 3 , 4). Ruthenium red staining confirmed these results.

An interesting and revealing finding was that there are identical staining reactions associated with the surface membrane of the epithelial cells of the conjunctiva (Figs. 3, 4). Apart from a few ribosomes within the epithelial cell cytoplasm these were the only two sites in the conjunctival epithelium where such a staining reaction took place.

Closer examination revealed that the reaction product of the staining was associated with the innermost membrane layer of the vesicles and the outermost layer of the cell membrane (Fig. 2). This staining was not spread evenly throughout the membrane. It occurred in punctate masses separated by bits of unstained membrane (Figs. 3, 5). These staining sites were evenly spaced on the membrane surface. This observation suggests that the mucus glycoprotein of the subsurface vesicles and of the surface layer of the epithelial cells was bound to specific sites in the cell membrane. The distribution on the cell surface was similar to that on the inner wall of the vesicle membrane (Fig. 4).

In sections stained with Thiéry's method ${ }^{8}$ there were chains of silver granules extending from these binding sites. Similar chains were found after iron haematoxylin staining. Such chains were found extending both from the wall of the subsurface vesicle into the cavity of the vesicle (Fig. 4) and out from the surface membrane of the epithelial cell into the overlying layer of mucus that came from the conjunctival epithelial goblet cells (Fig. 4).

The chains of silver granules branched and spread out in three dimensions from the binding sites. Such branching chains increased the area covered by the side chains and the amount of chain in contact with the goblet cell mucus.

Vesicles with similar staining properties to the subsurface vesicles were found in close association with the channels of endoplasmic reticulum and Golgi complex. There appeared to be an increase in the amount of Golgi complex in those cells that had an increased number of subsurface vesicles. Such observations suggest that the vesicles and their contents were produced by the endoplasmic reticulum and modified by the Golgi complex before they achieved the subsurface site. There was a considerable array of cytoskeletal elements in the cytoplasm just below the exposed surface of the epithelial cells (Fig. 2). It was probably these elements that guided the vesicles to their position beneath the membrane and finally their fusion with the membrane. The cytoskeletal elements probably also supported the cell membrane and helped to disperse the load exerted upon it by the tethered mucus.

\section{Discussion}

The subsurface vesicles appear to be a source of mucus with a very specific function. The role of the subsurface vesicle mucus is to anchor the goblet cell mucus to the surface membranes of the epithelial cells covering the eye. The mucus the vesicles contain is bound to the vesicle membrane and has long chains. When the subsurface vesicles fuse with the cell membrane of the epithelial cells and become in-

Fig. 1 Low power electron micrograph showing a healthy cell in the outermost layer of the conjunctival epithelium that contains a layer of subsurface vesicles beneath its microvillous border. $(\times 5600)$.

Fig. 2 Details of the surface membrane, and subsurface vesicle membrane showing that the staining is confined to one leaflet of the unit membrane. $(\times 60000)$.

Fig. 3 Silver stained electron micrograph showing the similarity in staining properties between the subsurface vesicle contents and the masses associated with the microvilli. (×32 500).

Fig. 4 Electron micrograph showing the mucus glycoprotein chains extending out from the microvillous surface, as well as others within the subsurface vesicles. (×81 400).

Fig. 5 High-power electron micrograph showing that the distribution of stain on the microvillous surface is not uniform but is distributed in evenly spaced clumps. $(\times 75000)$.

Fig. 6 A diagram to illustrate the way that the subsurface vesicle fuses with the cell membrane and thus exposes its contents to the mucus secreted by the goblet cells.

Abbreviations:

$\mathrm{cs}=$ External surface of conjunctiva, the site of the tear film. $\mathrm{ds}=$ Densely staining anchor site, where the mucus glycoprotein is attached to the microvillous membrane. $\mathrm{f}=$ Filamentous cytoskeletal elements. $\mathrm{g}=$ Golgi apparatus. $\mathrm{gm}=$ Goblet cell mucus. $\mathrm{mc}=$ Mucous glycoprotein chain. $\mathrm{mv}=$ Microvillus. $\mathrm{n}=$ Nucleus. $\mathrm{sc}=$ Surface cell of the conjunctival epithelium. $\mathrm{sv}=$ Subsurface vesicle. 


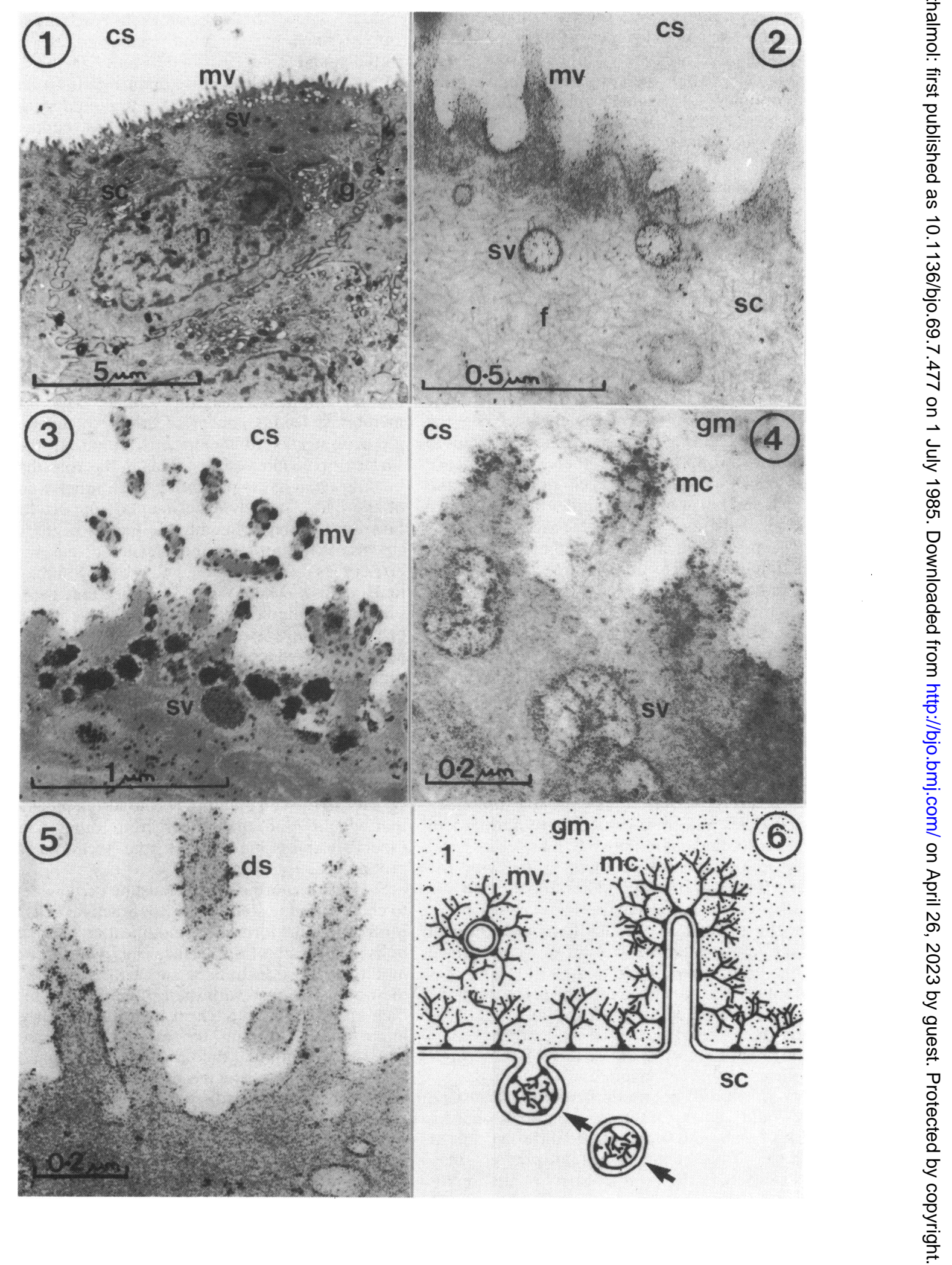


corporated into the cell surface, these chains extend outwards into the mucus layer covering of the surface (Fig. 6). This covering layer of mucus is secreted by the goblet cells within the conjunctival epithelium. The intermingling of the mucus glycoprotein chains with mucus mechanically and probably chemically bonds the mucus layer to the surface of the eye and increases its adhesion to the conjunctival surface. Any such increase in adhesion will resist the cohesive forces of the tear film and thus oppose break up of the tear film.

The increase in numbers of subsurface vesicles that occur in some external eye diseases may reflect an attempt to increase the binding of the mucus to the eye surface. There is of course the alternative explanation that these vesicles are inhibited in some way from discharging their contents and so have accumulated beneath the surface.

One can propose the hypothesis that there is some factor in normal tear secretions that acts on the superficial cells causing the submembrane vesicles to be released. In normal eyes there is a balance between vesicle release and vesicle production, and thus vesicles do not accumulate under the cell surface membrane. Whereas in the diseased state this balance is upset. Such an upset could either be in the composition of the tear film-say a reduction in the potency or amount of a vesicle releasing agent-or a change in the surface cells themselves. Evidence that such a mechanism might exist and the nature of the releasing agent comes from the work of Meier and Hay, ${ }^{9}$ who showed that there was stimulation of extracellular matrix synthesis in the developing cornea by glycosaminoglycans.

There is no doubt that in the diseased eyes I have examined some of the subsurface vesicles do fuse with the surface membrane and produce apparently normal anchoring chains. It is probable therefore that the vesicles and their contents are normal and that the fault in the anchoring system lies elsewhere, probably in the control of their fusing with the external cell membrane. In late keratoconjunctivitis sicca, however, the subsurface vesicles are absent.

When the subsurface vesicles fuse with the cell membrane, a considerable amount of extra surface membrane becomes available to the cell. This incorporated subsurface vesicle membrane is probably the source of the cell membrane of the microvilli that sprout on the exposed surface of the epithelial cells; microvilli are not found on the surfaces of the deeper cells in the epithelial cell layers.

The production of microvilli from the subsurface vesicles would also explain the varying sizes, shapes, and form of microvilli found on the differing cells at the surface. These changes in morphology may represent different stages in maturity of the micro- villi. It is known that when new cells reach the surface only a small part of their membrane is exposed initially, and that it bears a different size and density of microvilli from the more mature cells around it. ${ }^{10}$ This suggests that the process is triggered by the cell reaching the surface, and, since only a small area of cell surface is exposed initially, the demands upon the cell to produce microvilli will be less than if the whole surface were exposed at once.

The nature of the membrane binding site has not been determined. The molecular link might be the lipophilic part of the mucus glycoprotein. It is interesting to speculate, however, that they might be lectins. Lectins are carbohydrate-bonding proteins that have two critical properties, specificity for a particular sugar residue and bivalency or polyvalency. They are usually subdivided on the basis of whether or not they integrate into membranes. The non-membrane lectins are soluble. The integral membrane lectins appear to have evolved to bind glycoconjugates to membranes either at the cell surface or within vesicles. This is the role that the binding agent has to perform in the epithelial surface of the eye. There is experimental support for the function of integral membrane lectins in the endocytosis of glycoproteins and in cellular translocation. So perhaps it is not too much to assume a similar role in exocytosis. There is also evidence that the major function of soluble lectins is to bind the complementary glycoconjugates on and around the cells that release them. Since both the lectins and the glycoconjugates may be multivalent, large macromolecular complexes that shape the extracellular environments may be formed. Immunohistochemical studies suggest that there is secretion of soluble lectins."

We have here not only a mechanism that would bind that mucoprotein secreted by the subsurface vesicles to the cell membrane, but also a mechanism that would bind the mucus from other sources, especially the goblet cells, to the anchored mucoprotein.

Such an anchoring system must be very vulnerable to changes in the state of the conjunctival cells. Any form of squame formation, modification of the metabolism, or death of the surface cells would probably modify the production of subsurface vesicles and their ability to fuse with the cell membrane to fulfil their anchoring role. Such an anchoring role is vitally important in keeping the eye wet, because the cohesive force of tear fluid is greater than that of the naked conjunctiva, and it is the 10 or so dynes per $\mathrm{cm}$ contributed by these adhesive links that increase the adhesive forces of the conjunctival surface sufficiently to promote tear film spreading across the conjunctiva and to achieve all-over wetting of the surface vital for a healthy eye. 
The role of the surface microvilli and the binding chains is essentially similar: they both stop the most adjacent layer of mucus moving. It is probable that the turbulence induced by the microvilli will stop the flow of mucus around them, and the chains of mucus glycoprotein will extend their effect. Their effect will be that the tear flow will be much greater near the surface of the film than near its epithelial interface.

The role of the goblet cell mucus is probably one of lubricating the movement of the eyelid. And having its innermost layers anchored will ensure that the breaking of the mucus-layer shear forces induced by lid movements will occur well clear of the cell surface. This will protect the epithelial cells from damage by movements of the lid.

$A$ reduction in the effectiveness of subsurface vesicle mucus-glycoprotein binding, however caused, will reduce the adhesive forces holding the goblet cell mucus to the eye surface. Such a reduction will allow movement of the mucus, reduce the thickness protecting the epithelial cells, and ultimately contribute to a more rapid tear film break up.

I thank Mr Ian Mackic, FRCS, for enthusiastic support, Miss C Cope for dedicated technical expertise, and Mrs M Coulton for typing.

\section{References}

1 Wanko, T, Lloyd, BJ, Matthews J. The fine structure of human conjunctiva in the perilimbal zone. Invest Ophthalmol Visual Sci 1964; 3: 285-301.

2 Takakusaki I. Fine structure of the human palpebral conjunctiva with special reference to pathological changes in vernal conjunctivitis. Arch Histol Jpn 1969; 30: 247-82.

3 Srinivasan BD, Jakabiec FA, Iwamoto T, Devoe AG. Giant papillary conjunctivitis with ocular prosthesis. Arch Ophthalmol 1979; 97: 892-5.

4 Greiner JV, Henriquez AS, Weidman TA, et al. 'Second' mucus secretory system of the human conjunctiva. Invest Ophthalmol Visual Sci 1979; 18 (ARVO suppl): 123.

5 Greiner JV, Kenyon KR, Henriquez AS, Korb DR, Weidman TA, Allansmith MR. Mucus secretory vesicles in conjunctival epithelial cells of wearers of contact lenses. Arch Ophthalmol 1980; 98: 1843-6.

6 Greiner JV, Allansmith MR. Effects of contact lens wear on the human conjunctival mucus system. Ophthalmology (Rochester) 1981; 88: 821-32.

7 Dilly PN, Mackie IA. Surface changes in the anaesthetic conjunctiva in man, with special reference to the production of mucus from a non-goblet-cell source. Br J Ophthalmol 1981; 65: 833-42.

8 Thiéry J-P. Mise en évidence des polysaccharides sur coupes fines en microscopie électronique. J Microscopie 1967; 6: 987-1018.

9 Meier S, Hay ED. Stimulation of extracellular matrix synthesis in the developing cornea by glycosaminoglycans. Proc Natl Acad Sci USA 1974; 71: 2310-3.

10 Hoffman $F$. The surface of epithelial cells in the cornea under the scanning electron microscope. Ophthalmic Res 1972; 3: 207.

11 Barondes SH. Soluble lectins: a new class of extracellular proteins. Science 1984; 223: 1259-64. 